\title{
A NOTE ON THE HOMOLOGY OF DELETED PRODUCT SPACES
}

\author{
C. W. PATTY
}

If $X$ is a topological space, let $D_{X}$ denote the subset of $X \times X$ consisting of the set of all points of the form $(x, x)$, where $x \in X$. Then the deleted product space, $X^{*}$, of $X$ is the space $X \times X-D_{X}$ with the relative topology. The purpose of this note is to prove the following theorem.

Theorem. Let $Z$ denote the group of integers. If $X$ is a finite, $n$ dimensional polyhedron such that $H_{n}(X, Z)=0$, then $H_{2 n}\left(X^{*}, Z\right)=0$.

If $X$ is a finite polyhedron, let

$$
P\left(X^{*}\right)=U\{r \times s \mid r \text { and } s \text { are simplexes of } X \text { and } r \cap s=\varnothing\} \text {. }
$$

It is shown in [1] that if $X$ is a finite polyhedron, then there is a deformation retraction of $X^{*}$ onto $P\left(X^{*}\right)$. If $X$ is a finite polyhedron, let $X^{\prime}=U\left\{r \times s \mid r\right.$ and $s$ are faces of simplexes $r^{\prime}$ and $s^{\prime}$ of $X$ such that $\left.r^{\prime} \cap s^{\prime} \neq \varnothing\right\}$.

LEmma. If $X$ is a finite, n-dimensional polyhedron, then $P\left(X^{*}\right) \cap X^{\prime}$ has dimension $\leqq 2 n-1$.

Proof. Let $\sigma$ be a cell of $P\left(X^{*}\right) \cap X^{\prime}$. Since $\sigma$ is a cell of $P\left(X^{*}\right)$, $\sigma=r \times s$, where $r$ and $s$ are simplexes of $X$ such that $r \cap s=\varnothing$. Since $\sigma$ is a cell of $X^{\prime}, r$ and $s$ are faces of simplexes $r^{\prime}$ and $s^{\prime}$ such that $r^{\prime} \cap s^{\prime} \neq \varnothing$. Therefore, either $r$ is a proper face of $r^{\prime}$ or $s$ is a proper face of $s^{\prime}$. Hence $r \times s$ has dimension $\leqq 2 n-1$.

Proof of the Theorem. Now $\left(X \times X ; P\left(X^{*}\right), X^{\prime}\right)$ is a proper triad. Consider the Mayer-Vietoris sequence of this proper triad.

$$
\begin{aligned}
\leftarrow & H_{2 n}\left(P\left(X^{*}\right), Z\right)+H_{2 n}\left(X^{\prime}, Z\right) \leftarrow H_{2 n}\left(P\left(X^{*}\right) \cap X^{\prime}, Z\right) \leftarrow 0 \\
& \quad \ldots \leftarrow H_{2 n}(X \times X, Z) .
\end{aligned}
$$

By the lemma, $H_{2 n}\left(P\left(X^{*}\right) \cap X^{\prime}, Z\right)=0$, and $H_{2 n}(X \times X, Z)=0$ by the Künneth formula. Therefore, by exactness of the Mayer-Vietoris sequence, $H_{2 n}\left(P\left(X^{*}\right), Z\right)=0$.

\section{REFERENCE}

1. A. Shapiro, Obstructions to the imbedding of a complex in a euclidean space. I. The first obstruction, Ann. of Math. (2) 66 (1957), 256-269.

University of North Carolina

Received by the editors July 30, 1962. 